\title{
Studying Gender Diversity
}

Jennifer D. Rubin $\dagger^{* 1}$, S. Atwood $\dagger^{1}$, and Kristina R. Olson ${ }^{1}$

${ }^{1}$ Department of Psychology, University of Washington, Seattle, WA 98105 USA.

*Correspondence: jdrubin@uw.edu. (J.D. Rubin)

tThese authors contributed equally.

Keywords: gender identity, gender diversity, gender nonconformity, gender development, gender cognition, attitudes

\footnotetext{
Abstract

Gender identity is a core feature of human experience, yet our understanding of gender identity is shifting with broader societal changes in recognizing and understanding gender diversity. Here we discuss recent trends and upcoming directions for this burgeoning subfield.
} 
Within the last decade in many Western cultures there has been increasing recognition of gender diversity (see Box 1, Table I for definitions of all bolded words)-including people who are transgender, nonbinary and others-in popular media, medicine, and the legal sphere. This is a departure from the traditional view which conceives of gender as a binary and discrete category (e.g., you are a boy or a girl), and is typically assumed to align with one's assigned sex. However, emerging evidence indicates that gender may be more complex than these early conceptions. Further, research suggests that the numbers of people identifying as gender diverse may be rising [1] with recent population-based samples of adolescents suggest that $1 \%$ to nearly $3 \%$ of young people identify as gender diverse (e.g., transgender, genderqueer; $[2,3]$ ). Examples of the greater societal recognition of gender diversity include increased legal recognition of nonbinary identities in several countries (e.g., parts or all of Australia, Denmark, United States, Argentina), the addition of singular 'they' in Merriam Webster dictionaries, and the first transgender superhero on U.S. television. Yet, psychology research on gender diversity lags behind.

In this review we summarize research developments in the last 5 years on the psychology of gender diversity. We focus on three areas where advances have primarily been made: large descriptive studies documenting the experiences of gender diverse people, theoretical and empirical work advancing the measurement of gender diversity, and experimental studies of people's perceptions of and attitudes toward gender diverse people (a fourth, work on mental health, appears in brief in Box 2). Finally, we discuss what we see as necessary next steps in research on gender diversity, An extended list of references listed by subtopic can be found in the Supplementary Materials.

\section{Documenting the experiences of gender diverse people}

The first area in which researchers have made progress in expanding our understanding of gender diversity is by documenting the lived experiences of gender diverse people in large-scale studies. Until the last decade, most documentation of gender diversity came from media exemplars (e.g., 
Christine Jorgenson in the 1950's), autobiographies (e.g., Janet Mock, Kate Bornstein), and within the research domain, qualitative interviews (e.g., [4]).

More recently, quantitative approaches have investigated the experiences of gender diverse people, including the largest study to date, that surveyed over 27,00o gender diverse adults [5]. Among the major findings were: almost half of gender diverse adults experienced anti-transgender harassment in the previous year, family rejection was associated with suicidality (and other negative indicators of well-being), and transgender people showed large economic disparities (e.g., living in extreme poverty; higher unemployment rates) compared to the U.S. population. Despite these hardships, respondents also indicated positive changes, such as growing visibility and acceptance of gender diverse people in the U.S.

In parallel to studies of gender diverse adults, quantitative researchers have begun to document the lives of transgender and gender nonconforming children, who are for the first time in Western cultures, increasingly being affirmed in their gender expressions during early childhood. These children differ dramatically from the adults mentioned above, in having greater support from their families and the option to transition socially and medically earlier than past cohorts of transgender people. Overall, these studies have found that the gender development of young transgender children who are affirmed in their identities looks remarkably similar to that of their cisgender peers [6]. Additional opportunities for developmental research with gender diverse populations include understanding the various pathways to gender diverse identities, including the trajectories of individuals with less binary identities, and individuals who first identify as transgender later in development.

\section{Measuring gender identity in psychological science}

A second area of focus in recent work on gender diversity has been the theoretical and empirical work on the measurement of gender identity and related constructs. Critically, the previous measures often cannot be used with more gender diverse samples as they fail to account for the full range of 
gender experiences, resulting in unintentional missing data for those people who do not identify as boys

or girls (or men and women [7]). Moreover, they frequently contain questions that are worded in ways that make it a challenge for gender diverse people to understand which dimension of their identity is being assessed (e.g., sex versus gender), likely leading to some mis-classification (e.g., [7]).

Recent work has improved upon past measures by developing multi-question items to assess identity, recognizing that there are often multiple components of gender identity (e.g., [7,8]; see also Box 1 for an example measure). Though these new measures were often initially designed to better document the experiences of gender diverse people, their use in wider samples has led us to realize that cisgender people often see their gender as less binary and more multidimensional than former measures suggested. For instance, a recent study investigating gender identity in a large sample of children found that more than half reported similarity to multiple gender groups (e.g., boys and girls; [8]). For this reason, we and other researchers believe that free responses and less categorical measures have the potential to expand our understanding of gender in both gender minority and gender majority populations [9]. As language and identity continues to shift, this line of work is likely to continue to develop in parallel to larger social changes to ensure that our measures best capture how people are thinking about their identities.

\section{Assessing and modifying attitudes towards gender nonconformity}

A third area of work related to gender diversity has examined cisgender people's perceptions of and attitudes toward gender diverse people. Large-scale national surveys suggest that attitudes toward transgender people are improving and that people are increasingly perceiving discrimination against transgender people as problematic [10]. One reason adults' attitudes may be shifting is that more gender diverse people are coming out [5]. The resultant exposure, recognition, and knowledge about gender minorities may be playing a role in reducing levels of transphobia and greater acceptance of transgender people [11]. 
Additionally, some studies have begun testing effective strategies for attitude change about gender diversity. In perhaps the most rigorous study on the topic, canvassers engaged voters in brief perspective-taking conversations about transgender people's rights. These conversations led to support for a nondiscrimination law protecting transgender people's rights and long-term decreases in transphobia [12], providing some of the first causal tests of attitude change regarding transgender people.

Other work in this area examines associations with and predictors of negative attitudes toward transgender people in both perceivers (e.g., masculine self-identification, gender essentialism; [13]) and targets (e.g., androgyny; [14]). However, much of the work to date is exploratory and raises further questions about when and why people have less favorable attitudes toward gender diverse people as well as the downstream implications of these beliefs on the lives of gender minorities. We see a clear opportunity for more work in these areas, particularly with larger, well-powered studies to understand the causal mechanisms behind attitudes toward and beliefs about gender diverse people.

\section{Conclusions and next steps}

A broad goal of cognitive science is to advance fundamental knowledge about human cognition and to generate theories about how humans perceive and conceptualize the world. Yet, in order to accurately do so, it is important to capture the full range of variation in human experience. Until very recently, the focus of most studies of gender identity and gender roles was on the experiences of cisgender people. The resulting theories failed to account for the experiences of individuals who are transgender, nonbinary, or otherwise do not reside within discrete gender categories. Thus, a newer movement toward documenting basic descriptive information-such as how transgender and nonbinary people identify their gender-is an important first step to eventually developing more inclusive and upto-date models about identity, categorization, and the mental representation of gender. In this process 
we encourage the increased inclusion of gender diverse people not only as participants, but as researchers.

In addition to advancing theory, research on human gender diversity is likely to lead to more applied contexts and policy prescriptions. For example, we can begin to investigate when and why transgender and nonbinary people of color experience disproportionate rates of discrimination and violence in their everyday lives [5] and intervene upon both the individual and structural factors contributing to these disparities. Further, it's important to examine the impact of state and federal legislation on the health of gender diverse people [15]. In the U.S. for example, state laws prohibit many gender diverse people from using public restrooms that align with their gender identity. Future research should examine if these social environments contribute to negative health outcomes and heightened rates of mistreatment for gender diverse people.

Ultimately, our hope is that the work summarized here, as well as the future work in this area, can simultaneously enhance our understanding of gender diversity, lead to the development of better measures, inform our understanding of the mechanisms underlying prejudice and discrimination, and ultimately improve the lives of gender diverse people and their loved ones. We expect the findings of the last 5 years to be only the tip of a much larger iceberg in which our understandings of gender and related constructs continue to expand and improve. 


\section{Acknowledgements}

We would like to thank Zach Schudson, Will Beischel, and Selin Gülgöz for their comments on previous drafts of this paper. 


\section{Box 1: Measuring Gender Diversity}

In the last few years, researchers have developed questions to assess gender diversity (see Table I for definitions of all bolded terms). Multi-question items, such as the example below, capture three dimensions of gender/sex: birth assigned sex, current gender identity, and transgender status (through cross-classification).

This first question assesses gender identity:

$\underline{\text { I identify as (a)... }}$

○ Male/Man

- Female/Woman

$\circ$ Non-binary

- Gender not listed here. Please specify

This second question assesses assigned sex:

On my original birth certificate, my sex is listed as:

○ Male

$\circ$ Female

- Another sex or classification. Please specify

Researchers can examine responses across the two questions to determine if someone is cisgender or transgender.

Table I. Definitions of Key Terms

\begin{tabular}{|l|l|}
\hline Term & Definition \\
\hline Gender diversity & $\begin{array}{l}\text { We use this term to refer to people with gender identities that do not coincide with } \\
\text { their birth assigned sex. Examples include nonbinary, gender non-conforming, and } \\
\text { transgender people. }\end{array}$ \\
\hline Gender identity & A label used to describe one's identity within a given society's understanding of gender. \\
\hline Non-binary & $\begin{array}{l}\text { A person who does not subscribe to conventional gender categories but identifies with } \\
\text { neither, both, or a combination of genders. Related terms include genderqueer, gender } \\
\text { fluid, and more. }\end{array}$ \\
\hline Assigned Sex & $\begin{array}{l}\text { The determination made at a person's birth based on an infant's anatomy and/or sex } \\
\text { chromosomes. }\end{array}$ \\
\hline Cisgender & $\begin{array}{l}\text { When one's gender identity and assigned sex coincide with societal norms (e.g., man } \\
\text { and male). }\end{array}$ \\
\hline Transgender & $\begin{array}{l}\text { When gender identity and sex do not coincide with societal norms (e.g., man and } \\
\text { female, sometimes this term is used to include nonbinary people). }\end{array}$ \\
\hline
\end{tabular}




\section{Box 2: Overview of Clinical Psychology \& Gender Diversity}

While research on gender diversity has a longer history within clinical psychology and psychiatry than within other branches of psychology, much of the early research originated from a medical model that viewed diversity of gender as a form of mental illness. Accordingly, "Gender Identity Disorder" made its first appearance in the Diagnostic and Statistical Manual in 1980, reflecting this view. Controversially, in 2013 it was renamed "Gender Dysphoria" to reflect the distress some people feel about their "cross-gender" identification and behavior.

In the last 5-10 years, views in clinical psychology have begun shifting to acknowledge the roles of minority stress, discrimination, and social rejection in explaining the association between gender diverse identities and mental health conditions such as anxiety, depression, suicidality, and eating disorders. Simultaneously, availability and access to gender affirming health care resources (e.g., hormones, surgeries, supportive therapists) has increased. Therefore, emerging research has examined the well-being of individuals who are affirmed in their identities, focusing on when and how this affirmation (e.g., through family support, state law changes, social transitions, medical transitions, etc.) does and does not contribute to well-being. Reading on these topics is included in the Supplemental Information. 


\section{References}

1 Wilson, B.D.M. et al. (2017) Characteristics and mental health of gender nonconforming adolescents in California: Findings from the 2015-2016 California Health Interview Survey, The Williams Institute and UCLA Center for Health Policy Research.

2 Perez-Brumer, A. et al. (2017) Prevalence and Correlates of Suicidal Ideation Among Transgender Youth in California: Findings From a Representative, Population-Based Sample of High School Students. J. Am. Acad. Child Adolesc. Psychiatry 56, 739-746

3 Rider, G.N. et al. (2018) Health and Care Utilization of Transgender and Gender Nonconforming Youth: A Population-Based Study. Pediatrics 141, e20171683

4 Halberstam, J. (1998) Transgender Butch: Butch/FTM Border Wars and the Masculine Continuum. GLQ J. Lesbian Gay Stud. 4, 287-310

5 James, S. et al. (2016) The report of the 2015 US transgender survey, National Center for Transgender Equality.

6 Gülgöz, S. et al. (2019) Similarity in transgender and cisgender children's gender development. Proc. Natl. Acad. Sci. 116, 24480-24485

7 Tate, C.C. et al. (2013) A Two-Question Method for Assessing Gender Categories in the Social and Medical Sciences. J. Sex Res. 50, 767-776

8 Martin, C.L. et al. (2017) A Dual Identity Approach for Conceptualizing and Measuring Children's Gender Identity. Child Dev. 88, 167-182

9 Morgenroth, T. and Ryan, M.K. (accepted) The effects of gender trouble: An integrative theoretical framework of the perpetuation and disruption of the gender/sex binary. Perspect. Psychol. Sci.

10 Flores, A.R. et al. (2016) Public Support for Transgender Rights: A Twenty-three Country Survey, Williams Institute, UCLA School of Law.

11 Barbir, L.A. et al. (2017) Friendship, attitudes, and behavioral intentions of cisgender heterosexuals 
toward transgender individuals. J. Gay Lesbian Ment. Health 21, 154-170

12 Broockman, D. and Kalla, J. (2016) Durably reducing transphobia: A field experiment on door-todoor canvassing. Science 352, 220-224

13 Harrison, B.F. and Michelson, M.R. (2019) Gender, Masculinity Threat, and Support for Transgender Rights: An Experimental Study. Sex Roles 80, 63-75

14 Stern, C. and Rule, N.O. (2018) Physical Androgyny and Categorization Difficulty Shape Political Conservatives' Attitudes Toward Transgender People. Soc. Psychol. Personal. Sci. 9, 24-31

15 Perez-Brumer, A. et al. (2015) Individual- and Structural-Level Risk Factors for Suicide Attempts Among Transgender Adults. Behav. Med. 41, 164-171 